\title{
A Novel Device for Self-acquisition of ECG Signal in Telemedicine Systems for Chronic Patients
}

\author{
Donati M., Benini A., Celli A., Iacopetti F., Fanucci L. \\ Dept. of Information Engineering \\ University of Pisa \\ Pisa, Italy
}

\author{
Fanucci L. \\ IngeniArs s.r.l. \\ Pisa, Italy
}

\begin{abstract}
The increasing diffusion of chronic diseases represents a challenge for the health systems that have to deal with rising costs caused by the large number of patients. The ICT-enabled telemedicine systems represent a promising solution to provide effective treatments, to reduce hospitalizations and related costs and to improve the patients' quality of life. These systems allow to monitor the clinical status of the patients through self-measured vital signs. The electrocardiogram (ECG) represents one of the most practiced clinical exams in the treatment of chronic patients, but ECG devices currently known for medical purpose are difficult to be used by non-skilled persons, and they are definitively not suitable for such telemedicine systems. This paper introduces a user-friendly and ergonomic device conceived to be used autonomously by the patient to record high quality ECGs. The device acquires the first lead of the ECG from the patient's hands exploiting dry metal electrodes. The device requires few basic actions to be operated and can transmit collected data via Bluetooth connectivity. The developed dedicated API makes also possible an easy integration of the device into telemedicine systems.
\end{abstract}

Keywords—ECG; EKG; Telemedicine; Medical Device

\section{INTRODUCTION}

In recent years, the management of cardiac, pulmonary and metabolic chronic diseases is becoming more and more challenging, both from the organizational and economical point of view. The total number of patients affected by such diseases has been increasing worldwide and this positive trend is expected to rise in the future. This is mainly due to the general ageing of the population and the advancements of the medical science that have improved the survival rate and the life expectation of these patients. For these reasons, the traditional chronic care model, which includes in-hospital care of the acute phases and out-of-hospital follow-up based on periodical check-ups, is becoming unsustainable for the health systems.

Among chronic diseases, heart failure (HF) represents a major public health issue due to its diffusion and high management costs [1]. According to the European Society of Cardiology (ESC), there are at least 15 million citizens suffering from $\mathrm{HF}$ and the prevalence is between $2 \%$ and $3 \%$ of the population [2] while the American Heart Association reports that more than 5 million Americans have HF, with a prevalence of $2.2 \%$ [3]. The incidences are 3.6 million and 550,000 new cases per year respectively in Europe and in U.S. [4]. The number of chronic HF patients is projected to dramatically increase by $50 \%$ in the next 20 years [5]. HF is the leading cause of hospitalization in Europe and U.S. [6], representing the $5 \%$ of acute hospital admissions [2]. Moreover, $\mathrm{HF}$ has a quite high re-admission rate, ranging between $25 \%$ and $30 \%$ at 3 months and reaching $50 \%$ at 6 months after discharge [1]. From the management point of view, the hospitalizations represent the costliest part of the economic burden sustained by the health systems [7], but also the post-acute follow-up visits account in significant way the resources of the health systems.

There are in literature evidences that multi-disciplinary management approaches including post-discharge in-home care with ICT-enabled telemedicine systems result in a reduction of the mortality, hospital admission rate and length of hospital stay [8]. According to recent review studies [9][10], the most effective telemedicine systems allow the patient to selfmeasure vital signs at home and to make them available for the medical personnel in charge of his/her care (i.e. clinicians, physicians and practitioners) without time and distance barriers. With this frequent assessment of the clinical status, otherwise impossible with the traditional care model, aggravations can be early detected and acted upon, avoiding a large part of the hospitalizations. This produces a reduction of the costs, a better scheduling of the resources and the enhancement of the patient's quality of life.

The ECG signal is one to the most useful vital sign to be monitored, especially in cardiac diseases like HF, being a direct measure of the heart activity. The ESC guidelines [2] recommend the monitoring of the ECG signal, while they consider weight, blood pressure, oxygen saturation and their trends over time an added value for a better assessment of the clinical status. Unfortunately, medical ECG devices currently known are not conceived for self-measurement performed by the patient. In fact, they require the correct placement of recording electrodes on the body to obtain a high quality ECG, and they often present complex human-machine interfaces and measuring methods not suitable for common people.

This paper presents an innovative ECG device enabling the patient to collect autonomously a high quality first lead ECG for telemedicine purpose, in a very simple way and without electrodes on the body. It features Bluetooth connectivity and an API for the integration in existing telemedicine systems. Hereafter, Section II describes related work. Section III presents the feasibility study of the device. Section IV and Section $\mathrm{V}$ present respectively the implementation and the testing results. Section VI presents the communication API. Finally, conclusions are drawn in Section VII. 


\section{RELATED WORK}

The state-of-the-art ECG devices suitable for remote monitoring of patients in telemedicine services, provided with Bluetooth connectivity to send data to telemedicine gateways, can be classified according to the target use. Two main categories exist: professional devices used by medical personnel and self-measurement devices usable directly by patients. Moreover, different measuring methods can be found in the ECG devices landscape.

The professional devices, such as Cardioline Microtel BT [11] and Corscience COR12 [12], provide high quality complete ECG for diagnosis purposes, and may also be used in telemedicine applications. They need the placement of a number of wet adhesive electrodes, ranging from four to ten, on specific parts of the body. The correct placement of the electrodes requires specific skills, resulting very difficult and often impossible for non-skilled users.

Conversely, the self-measurement devices are conceived for the autonomous use by patients and generally they are able to acquire less detailed ECG recordings than a professional device; it means that usually for a complete assessment of the heart activity and diagnosis, a professional device is further needed. Some devices (e.g. Intelesens V-Patch [13], Iansys Lifetouch [14] or Lifewatch Lifestar ACT [15]) use two, three or four adhesive wet electrodes to be placed on the body. A different technique, used by Card Guard PMP4 SelfCheck ECG [16], exploits two or more dry electrodes located on the device to be put and maintained in contact with specific areas of the body (e.g. the chest, etc.). Finally, in other devices such as DOCOBO doc@home [17] and HeartCheck [18], up to four dry electrodes are embedded on the device surface and the user has to place hands or fingers on the device or to grab it in a specific way.

\section{PRELIMINARy Studies}

In order to allow patients to record their own ECG signal, the sensor device has to be easy to use and ergonomic, providing an intuitive measuring method and minimizing the actions required to perform the acquisition. An important issue of current devices is that their measuring method relies on the placement of electrodes on specific areas of the body.

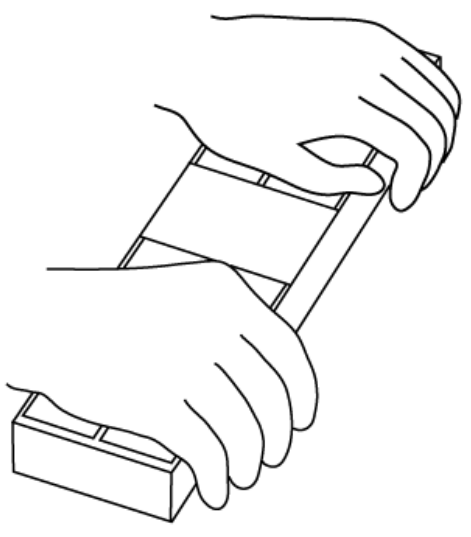

Fig 1. Selected ECG device shape and measuring method.

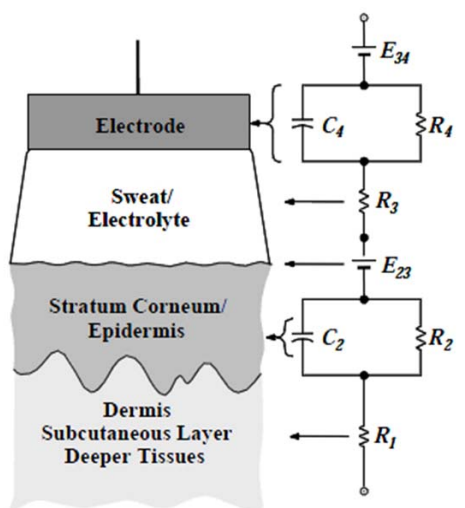

Fig 2. Electric model of the electrode-skin interface [20].

A solution to simplify the acquisition process consists of using dry metal electrodes installed on the device surface, on which the patient can rest the hands and maintain the contact for the duration of the acquisition. Nevertheless, such solution introduces some challenges due to the poor quality of the electrical contact between the metal electrodes and the skin that can produce artifacts on the ECG recording or even prevent the signal acquisition. Thus, studies on the device shape, the handelectrodes configuration and the electrode-skin impedance were carried out in order to assess the feasibility of the proposed solution.

\section{A. Device Shape and Electrode-skin Contacts}

The ergonomic shape of the device, that favours a comfortable position of the patient, is important in order to minimize the movements of the hands on the electrodes during the recording of the ECG signal. Moreover, the part of the hand that realizes the contact with the electrode is a determining factor in order to improve the contact quality. As reported in [19], a complete study about the shape of the device, the position of the electrodes and the arrangement of the contact with the hands was made. By placing the hands in contact with the defibrillation paddles of a professional ECG monitor, different hands-electrodes configurations were investigated and the quality of the signals detected were compared. Afterwards, several different shapes and ways to handle them were experimented involving some old people, in order to identify the best configuration. The selected solution, shown in Fig. 1, is a parallelepiped having approximated dimensions of $30 \mathrm{~cm} \mathrm{x}$ $5 \mathrm{~cm} \times 3 \mathrm{~cm}$ with the electrodes placed on the upper wider surface. This configuration allows to maintain easily the hands in contact with the electrodes for the duration of the measurement, minimizing also the residual movement of the hands because it helps to relax the muscles.

\section{B. Electrode-skin Impedance}

Fig. 2 represents a simplified model of the skin-electrode impedance that presents resistive and capacitive components [20]. The impedances of the two electrode-skin interfaces represent the source impedances in the measurement equivalent circuit. When a differential amplifier is used, unbalanced source impedances transform an amount of the common mode signal into a differential signal, degrading the Common Mode Rejection Ratio (CMRR) of the system. Due to the half-cell 


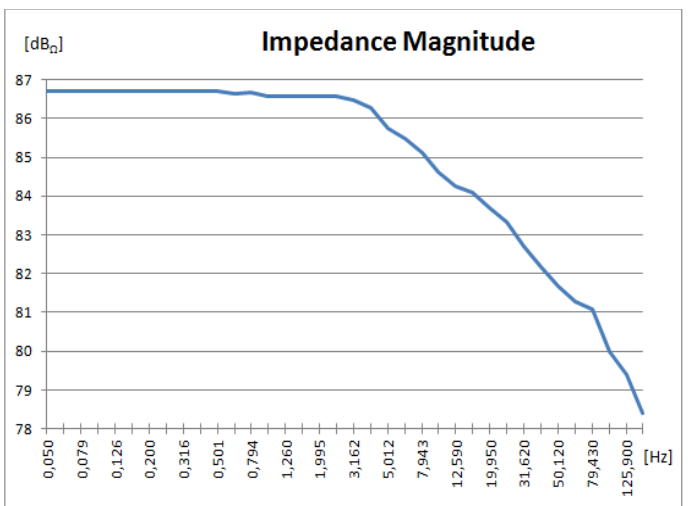

Fig 3. Graphic of the measured impedance magnitude.

potential of the skin-metal interface, a large common mode constant voltage, ranging from $300 \mathrm{mV}$ to $1 \mathrm{~V}$, is present at the inputs of the amplifier. Thus, a variable imbalance of the input impedances caused by the movements of the hands on the dry electrodes produces a low-frequency differential signal. This signal introduces artifacts on the recorded ECG and it can lead the input stage to saturation. The amplitude of the disturbing signal is proportional to the absolute value of the difference between the source impedances. When using dry metal electrodes it is impossible to guarantee two identical and constant contact impedances during the ECG recording. In order to minimize the amplitude of the disturbing signal, small input impedances are desirable. Moreover, the presence of a capacitive component of the skin-metal impedance introduces a phase distortion in the recorded ECG if the magnitude of the contact impedances are not negligible with respect to the input differential impedance of the amplifier. Thus, it is important to investigate the properties of the interface impedance in the point of the body where the signal is collected.

As reported in [19], a frequency characterization of the electrode-hand impedance was made, using dry steel electrodes placed in contact with each hand and fixed with elastic bandage to keep a constant contact pressure. A voltage-to-current converter and a voltage amplifier were designed and realized for this purpose. The input of the voltage-to-current converter was connected to a sinusoidal signal generator. The current was imposed on one of the electrodes, connected also to the voltage amplifier, while the other electrode was connected to ground. The output of the voltage amplifier was measured and shown with an oscilloscope. By comparing the input voltage amplitude with the output of the circuit at different frequencies it was possible to obtain the magnitude of the sum of the two contact impedances. Assuming that the impedances of the two interfaces are approximately equal, the value of a single contact impedance is half the measured value. As showed in Fig. 3, the maximum value of the impedance, measured in a frequency range including the band of the ECG signal, is at low frequencies and is equal to about $11 \mathrm{k} \Omega$. This result shows that the impedance magnitude is low enough to be considered negligible with respect to the input impedance of the amplifiers, that usually is much greater than $1 \mathrm{M} \Omega$. Thus, no further investigations about the phase of the impedance was made, since the capacitive component effects could be considered negligible.

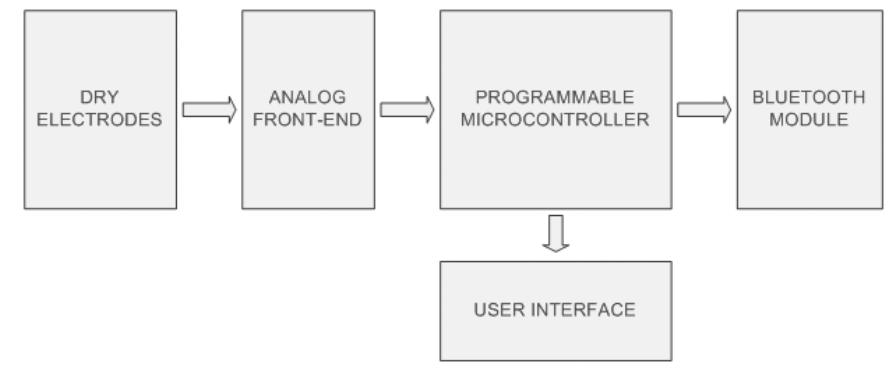

Fig 4. Block diagram of the ECG device.

\section{DEVICE IMPLEMENTATION}

A prototype of the proposed ECG device was realized implementing the selected shape. The plastic case of the device was designed using a 3D CAD software and realized with the use of a 3D-printer. Fig. 4 shows the block diagram of the prototype. The device consists of an analog front-end circuit that amplifies the signal collected by the electrodes and of a microcontroller unit that elaborates the signal and controls the user interface and the Bluetooth communication to transmit the recorded data.

\section{A. Dry Metal Electrodes}

The electric contact between the acquisition circuit and the patient's hands is achieved with the use of two pairs of stainless steel plates $(10 \mathrm{~cm} \times 2 \mathrm{~cm})$ placed on the upper surface of the plastic case. Each pair is kept in contact with a hand of the patient and includes a signal electrode, used to acquire the ECG electrical signal, and a reference electrode, used for the common mode reference.

\section{B. Analog Front-end}

Fig. 5 shows the schematic circuit of the analog front-end. The input stage consists of an instrumentation amplifier (IA) set by external resistors to have a gain of about 6.5 . The gain of the first stage is set low in order to avoid the saturation of the amplifier caused by the high common mode input voltage due to the half-cell potential of the skin-metal interface, when unbalanced input impedances are present. A negative feedback

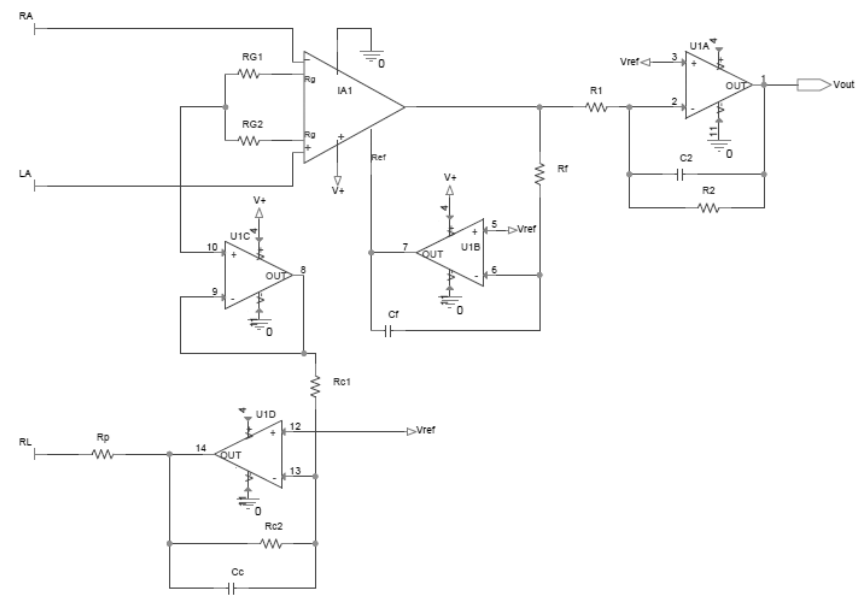

Fig 5. Schematic of the analog front-end. 


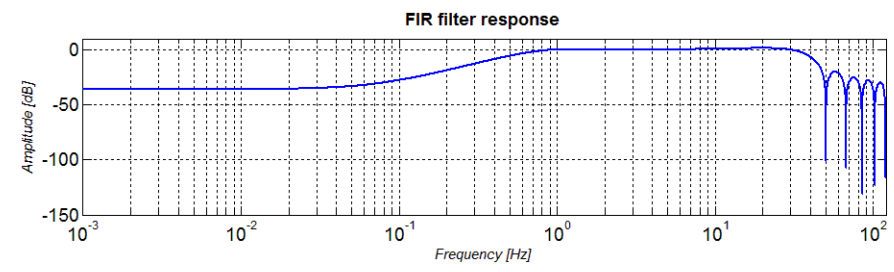

Fig 6. FIR filter amplitude response.

loop on the reference voltage of the IA, provided by an integrator, realizes a first-order high-pass response that filters out the DC offset and the low frequency components caused by the patient's movements. The single ended output of the first differential stage is then amplified by a gain stage that presents a single-pole low-pass response. The overall band of the analog front-end is $0.5 \mathrm{~Hz}-50 \mathrm{~Hz}$ and the total gain is set to 1000 . The common mode signal present at the inputs of the first stage is collected in the middle point of the gain resistance of the IA, it is then amplified, inverted and sent back to the body of the patient through the reference electrodes. This negative feedback loop realizes the so called "Right Leg Drive" that allows to reduce the amplitude of the common mode disturbing signal, caused by the coupling of the patients with the environmental sources of noise (e.g. the $50 \mathrm{~Hz}$ of the power network, etc.). Furthermore, by connecting one of the two inputs to the positive supply $\mathrm{V}+$ and the other to ground using two high value resistors (100 M 2 ), the output of the amplifier saturates when the electrodes are floating. This solution realizes a simple "lead-off detection" method that allows detecting if the electrodes are not in contact with the body. The output of the analog front-end is then supplied to an ADC channel of the microcontroller unit to be digitized and further processed.

\section{Digital Signal Processing}

The analog signal is digitized and processed by means of an Atmel SAM4L microcontroller featuring an ARM Cortex M4. The analog signal is converted by the embedded 12 bit ADC module with a sampling frequency of $500 \mathrm{~Hz}$, in accordance with the recommendations of the American Heart Association (AHA) guidelines [21]. After digitization, a high-selective linear-phase FIR filter with 941 coefficients is applied to the signal to achieve a clean ECG signal in a frequency range defined "monitoring band" suitable for ECG monitoring purposes [21]. As shown in Fig. 6, the FIR filter has a passband response ranging from $0.67 \mathrm{~Hz}$ to $37 \mathrm{~Hz}$ and a notch response that achieves an attenuation of $100 \mathrm{~dB}$ at $50 \mathrm{~Hz}$ (i.e. the frequency of the power network in Europe). This filter allows reducing the magnitude of the high frequency noise components and of the low frequency artifacts while preserving the main components of the ECG signal.

The heart rate (HR) is then calculated with an adaptive threshold-based algorithm applied to the energy of the ECG signal [22]. A high-pass filter with a cut-off frequency of $15 \mathrm{~Hz}$ is applied to the filtered ECG signal to highlight the QRS complex. The signal is then squared to obtain its power signal and the mean power is calculated with a moving window averaging operation. The peak-detection threshold is dynamically determined as the mean power multiplied by a constant value. When the power signal is greater than the threshold a new QRS complex is detected, and the HR is calculated as the inverse of the time between the current $R$ peak and its predecessor.

\section{Bluetooth Interface}

A Bluetooth 2.0 module with integrated antenna and Serial Port Profile (SPP) is used to transmit the samples of the ECG signal and the HR to a connected device, to be further visualized, elaborated or forwarded to remote telemedicine systems. The Bluetooth module is connected to the microcontroller through an UART data bus.

When the configuration button is pressed on the ECG device, it becomes discoverable for Bluetooth pairing and it can be paired with a remote device (e.g. a telemedicine gateway, etc.). This procedure is required only when pairing with a new remote device has to be performed. Once paired, the ECG device behaves as master and tries to open a connection with the paired gateway. When a connection is established and the hands are in contact with the electrodes, the device starts to record and send the ECG signal and the calculated HR to the gateway, which communicates with it by means of the ad-hoc designed API.

\section{E. User Interface}

Considering the target users, the user interface is intentionally very simple and consists of a display and of a couple of LEDs. Once powered, the device displays a message inviting the patient to put the hands in contact with the electrodes. Additionally, the battery status and the Bluetooth connection status are shown as well.

When contact is detected, the calculated HR is shown, a bar indicates the progress of the ECG transmission and a red LED blinks in correspondence of the R peaks. Fig. 7 shows the user interface of the device during a recording session.

\section{Testing Results}

The testing phase of the developed prototype had two main goals: testing the user-friendliness of the measurement method and verifying the quality of the acquired signal. The test was made using a computer acting as Bluetooth gateway and a Matlab® script to receive and plot the ECG signal data.

In order to test the device usability, 12 old people $(65-90$ years old) were asked to use the device and provide their feedbacks through a brief interview. Most testers did not use or did not have any particular confidence with home and personal electronic devices, e.g. smartphones, PCs etc.. The testers were

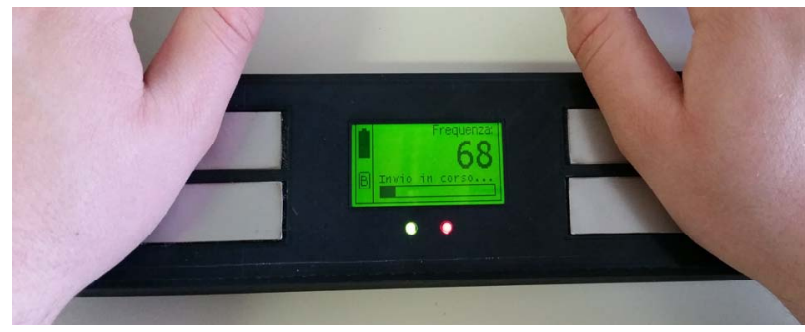

Fig 7. User interface of the device while recording an ECG. 
instructed on how to use the ECG device and they were asked to perform a series of two consecutive acquisitions. They were required to place and hold the hands on the electrodes, relaxing all the muscles of the arms until the ECG acquisition end. All testers were able to use the device and to successfully complete the acquisitions without any help. On specific request, all the testers substantially reported that it was easy for them to understand how to use the device and to perform the measurement. Additionally, they reported that the position of the hands is comfortable and easy to be maintained. The device usability will be anyway further investigated with the involvement of a larger number of potential users by means of a specific questionnaire.

In order to test the quality of the recorded ECG, the prototype output was compared with the ECG provided by a professional Lifepak 15 electrocardiograph configured for working in "monitor band" (i.e. $0.5 \mathrm{~Hz}-40 \mathrm{~Hz}$ ). The ECG signal of a tester was recorded using simultaneously both the prototype by placing the hands on its electrodes and the Lifepak 15 by attaching the wet electrodes on the chest. Fig. 8 shows the test configuration, the first lead of the ECG printed by the Lifepak 15 (black trace on the graph paper) and the ECG signal provided by the prototype (blue trace). A graphical comparison between the two signals shows that they are very similar and can be overlapped almost perfectly.

Further tests confirmed that the proposed device is able to provide high quality ECG signal acquisitions in "monitoring band" similar to professional equipment recordings, exploiting a very simple measurement method that overcomes the limits of the current ECG devices applied to first lead ECG selfacquisition performed by patients.
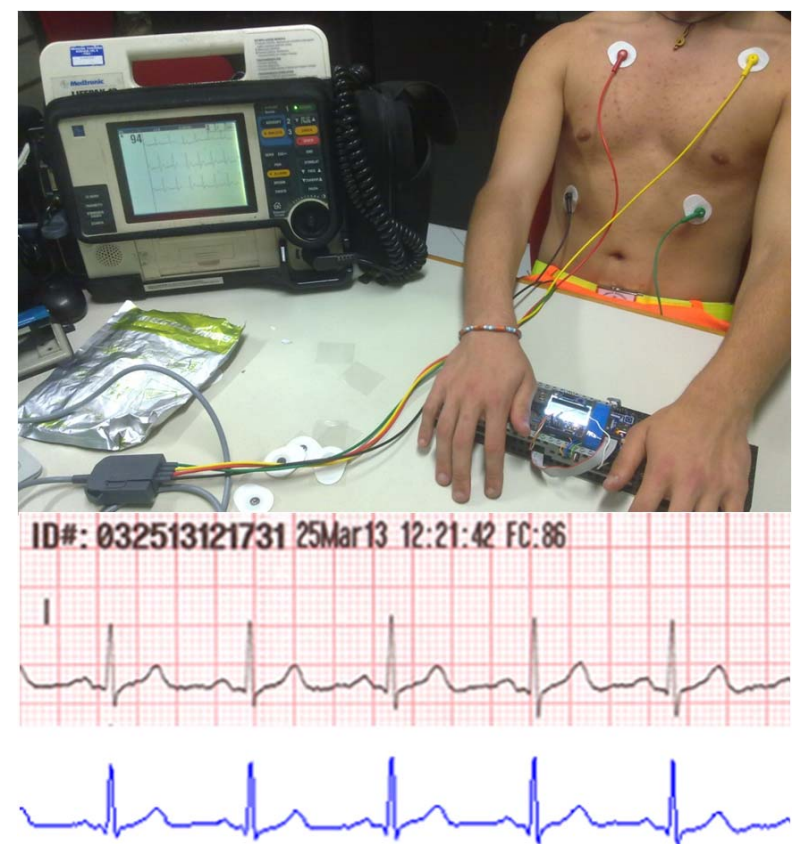

Fig 8. Comparison of the ECG acquired using Lifepak and the prototype.

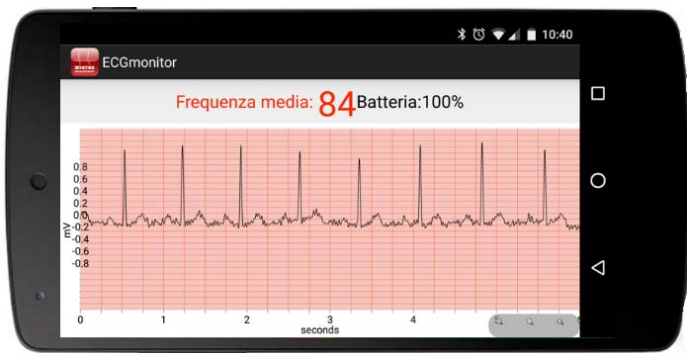

Fig 9. Test application running on an Android device.

\section{INTEGRATION WITH A TELEMEDICINE SYSTEM}

The purpose of the proposed ECG device is the use as a component of a wider telemedicine system in which an advanced gateway collects data from many different sensors according to the Wireless Sensors Network paradigm. The ECG device, once configured, is able to send, through a dedicated Bluetooth communication protocol, the ECG waveform, the hearth rate and the battery level. In detail, the device sends the waveform in real-time, the hearth-rate every 1 second and the battery level every 10 seconds.

In order to make the ECG device compatible and integrable with a large set of telemedicine gateways, a dedicated API was developed. This API allows the communication with the device implementing a set of functions and methods that covers all its functionalities. The essential functions to configure the gateway and communicate with the ECG device are:

- Pairing function: it tries to instantiate a Bluetooth connection with the ECG device to start the pairing procedure. Once the ECG device has been found, the pairing function provides for automatically sending the required PIN and complete the pairing phase.

- Configuration function: it allows to configure the duration of the ECG record.

- Acquisition function: it manages the data communication between the device and the gateway. The acquisition function waits for the packet "Ready to send" from the device and, once received, it sends a "Start" packet to trigger the device to start the ECG acquisition and the data transmission. The function also provides the method for handling the received packets and extracting the contained data.

- Stop-connection function: it provides the actions required to correctly close the connection between the gateway and the ECG device.

The API was tested realizing an Android application able to receive and show the ECG signal. Fig. 9 shows the application running on a LG Nexus 5 smartphone with Android 5.1 operating system.

\section{CONCLUSIONS}

The presented device allows the self-measurement of the first lead ECG for telemedicine purpose, enabling the patients to monitor frequently his/her heart activity without going to medical departments. Differently from the known professional 
devices used in medical departments, the device does not require to attach adhesive electrodes on the body of the patient, and it ensures a similar quality of the first lead of the ECG as obtained with professional devices configured in the monitoring band as specified by the American Heart Association [21]. Additionally, differently from other existing self-measurement devices that the patient has to grip or hold to allow an electric contact between the hands and the electrodes, the measurement method of the presented device avoids muscle tension, residual movements of arms and fingers and variations of the gripping force, allowing a high quality ECG. Finally, the developed API allows for an easy integration with existing telemedicine systems.

Future steps will investigate the possibility to add other dry electrodes on the bottom surface to create contacts also with the patient's legs in order to allow the measurement of more ECG leads. Moreover, a larger and structured testing campaign will be carried out on both usability and performance aspects. Finally, the device will be re-designed with particular attention to the design of hardware and firmware aimed at achieving the medical certification.

\section{REFERENCES}

[1] J. Parissis, et al, "Determinants of the direct cost of heart failure hospitalization in a public tertiary hospital", I. J. of Cardiology (2015)

[2] K. Dickstein, et al, "ESC guidelines for diagnosis and treatment of acute and chronic heart failure", European Heart J. (2008)

[3] A.S. Go, et all, "Heart disease and stroke statistics - 2014 Update, Circulation (2014)

[4] F. Zannad, et al, "Heart failure burden and therapy", Europace (2009)
[5] R.S. Gardner, "Chronic heart failure: epidemiology, investigation and management", Medicine (2014)

[6] A.P. Ambrosy, et al, "The global health and economic burden of hospitalization for heart failure", J. American cardiology (2014)

[7] W.C. Lee, Y.E. Chavez, T. Baker, B.R. Luce, Economic burden of heart failure: a summary of recent literature, Heart Lung 33 (2004)

[8] J. Fergenbaum, et al. "Care in the home for the management of chronic heart failure", Cardiovascular Nursing J. (2015)

[9] A.G. Ekeland, et al, "Effectiveness of telemedicine: a systematic revie of reviews", Int .J. of medical informatics (2010)

[10] P. Finet, et al, "Review of current telemedicine applications for chronic diseases", IRBM (2015)

[11] Cardioline Microtel BT, http://cardioline.com/prodotticardioline/microtel-bt, available at May 25, 2016.

[12] Corescience COR12, http://www.corscience.de/en/medicalengineering/products/ecg/cor12.html, available at May 25, 2016.

[13] Intelesens V-Patch Medical System, http://www.intelesens.com, available at May 25, 2013.

[14] Iansys Lifecare, http://www.iansys.com, available at May 25, 2016.

[15] LifeWatch, https://www.lifewatch.com/healthcare-professionals/lifestaract-ambulatory-cardiac-telemetry, available at May 25, 2016.

[16] Aura trade Portable handheld ECG, http://www.auratrade.sk/gb/products1/cm.html, available at May 25, 2016.

[17] Docobo, http://www.docobo.co.uk, available at May 25, 2016.

[18] HeartCheck, http://www.theheartcheck.com/, available at May 25, 2016.

[19] A. Benini, M. Donati, et al, "User-friendly Single-lead ECG Device for Home Telemonitoring Application", ISMICT, (2014)

[20] A. Baba, M.J. Burke, "Electrical characterisation of dry electrodes for ECG recordings," WSEAS (2008)

[21] P. Kligfield, et al, "Recommendations for the standardization and interpretation of electrocardiogram," Circulation (2007).

[22] J. Parak, J. Havlik, "ECG signal processing and heart rate frequency detection methods" Czech Tecnical University in Prague, unpublished. 